\title{
Follow-Up: The Evidence
}

\section{Domenico D'Ugo Alberto Biondi Andrea Tufo Roberto Persiani}

Department of Surgery, 'A. Gemelli' Hospital, Università Cattolica del Sacro Cuore, Rome, Italy

\section{Key Words}

Gastric cancer · Postoperative follow-up · Surveillance

\begin{abstract}
There is currently no consensus on the best strategy for the follow-up of patients who have undergone surgical treatment with curative intent for gastric cancer. The wide variation in recommendations for surveillance among international experts and hospital schedules clearly reflects a lack of an established body of evidence on this subject. Consequently, most of the international guidelines aimed at early detection of disease recurrence gloss over details concerning the mode, duration, and intensity of surveillance since they cannot be based on an acceptable grade of recommendation. Very few report anything other than the detection of recurrences or death as the primary endpoints, and, given the poor survival of patients with recurrent gastric cancer, the prognostic effect of early detection seems doubtful. In recent years, an increasing focus on evidence-based medicine, which has coincided with a growing concern about costs and efficiency in medicine, has caused a reevaluation of most surveillance practices. In this paper, we review and discuss the current body of evidence and follow-up practices after curative resection of gastric cancer.
\end{abstract}

Copyright $\odot 2013$ S. Karger AG, Basel

\section{KARGER}

E-Mail karger@karger.com

www.karger.com/dsu

\section{Introduction}

The potential value of a surveillance program in patients who have undergone cancer surgery is to detect recurrences in the early and asymptomatic period, to identify complications associated with surgery, and to collect outcome data. Early detection of cancer recurrence may be associated with improved survival because it may provide an opportunity for treatment to be initiated while the patient's condition is sufficiently stable to receive effective therapy.

A postoperative follow-up schedule is recommended for nearly all cancers in international guidelines even though the value of postoperative surveillance remains controversial $[1,2]$. In colorectal and breast cancers, several randomized controlled trials and meta-analyses have demonstrated an overall survival advantage associated with detection of recurrences through intensive followup as compared with patients who present later with symptomatic recurrences $[3,4]$.

Gastric cancer is one of the most frequent malignancies and the second leading cause of cancer deaths worldwide, with 989,600 new cases and 738,000 deaths in 2008, accounting for $8 \%$ of the total cases and $10 \%$ of total deaths for cancer [5]. 
Recurrence is the most important factor associated with death even after potentially curative gastrectomy. Over two thirds of recurrences occur in the first 3 years following surgery and fewer than $10 \%$ occur after 5 years; given the poor survival of patients with recurrent gastric cancer, only palliative therapy is generally possible [6-8].

Most clinicians perform postoperative surveillance for their gastric cancer patients during the first 3 years after surgery. However, there is no consensus on the most appropriate regimen and frequency of follow-up after curative surgery [9]. It must also be said that patients could be possibly reassured from regular follow-up, although the psychological benefit of surveillance is debatable. There is a wide variation in recommendations for surveillance among experts. In recent years an increasing focus on evidence-based medicine that has coincided with growing concern about costs and efficiency in medicine has caused a reevaluation of surveillance practices.

To date, all of the recommendations on surveillance are based on low-level evidence or no evidence at all due to the complete lack of randomized controlled trials on this peculiar subject. Very few report anything other than the detection of recurrences or death as the primary endpoints, and the prognostic effect of early detection seems doubtful given the poor survival of patients with an ascertained recurrent gastric cancer [9].

The objective of this study is to review the literature about the efficacy of follow-up protocols and investigations after gastrectomy for cancer. Moreover, in this study we have tried to classify different follow-up policies after curative resection for gastric cancer using a questionnaire-based survey among high-volume centers for the treatment of gastric cancer worldwide.

\section{Reasons for Follow-Up}

Surveillance after surgery in gastric cancer includes three main purposes: detecting local or distant recurrences and/or metachronous cancers in the remnant stomach, detecting long-term or late effects of surgical treatment, and collection of outcome data to evaluate the effectiveness of treatments and for research purposes.

\section{Recurrence}

The recurrence patterns of gastric cancer are classified as locoregional, peritoneal, and hematogenous. Locoregional recurrence is defined as cancer recurrence at the resection margin, within the lymph nodes (including regional, retropancreatic, retrocrural, and para-aortic nodes), or in the operation bed within the region of the resection (below the diaphragm and liver and above the pancreas and abdominal wound). In addition, the resection margin is divided into the proximal margin (including the lower third of the esophagus, remnant stomach, and gastrointestinal anastomosis) and the distal margin (duodenal stump). Peritoneal recurrence is defined as cancer recurrence in the abdominal cavity because of intraperitoneal distribution including visceral metastasis and rectal shelf, pericholedochal, and periureteral infiltration. Hematogenous recurrence has been defined as any metastatic lesion detected in distant organs [10, 11].

The timing of recurrence has been investigated by many researchers and data are not uniform as reported. More than $90 \%$ of patients relapse within 5 years after surgery and $70 \%$ relapse within $2-3$ years $[6,7]$. In early gastric cancer, the rate of recurrence after gastrectomy is reported to vary from 1.3 to $12.2 \%$. Median time to recurrence is 16 months, and hematogenous spread is probably the most common pattern of recurrence [12].

Many investigators have analyzed recurrence patterns, but the data have shown variable incidences of these patterns. This disagreement is attributed to differences in patient population, stage of the disease at the time of diagnosis, surgical treatment, and the mode and timing of recurrence detection. Based on a review of the literature, the pattern of recurrence tends to be local in the West, whereas the pattern is different in the East, with more peritoneal and hematogenous recurrences [9].

Occasionally, after partial gastrectomy a second primary tumor can arise in the remnant stomach. Much of the literature relates to gastrectomies for peptic ulcer disease, which estimates a risk that is not so different from the general population $[13,14]$.

As regards gastric cancer, second primaries are more common after surgery for early gastric cancer because these patients have a good prognosis after curative surgery. The reported incidence of metachronous gastric cancer after partial gastrectomy for early gastric cancer is $0.6-3 \%$ [15]. In clinical oncology practice, the detection of the recurrence in the early stage may provide an opportunity for effective treatment when patients are still fit enough to receive surgical or medical therapy.

Patients with gastric cancer recurrence are more often managed similarly to nonresectable patients because early detection of recurrence is quite difficult and peritoneal recurrence, one of the main patterns of recurrence, is usually diagnosed at an advanced stage. 
With the possible exception of a few locoregional (anastomotic and lymph nodal) and hepatic metastases suitable for radical resection, the vast majority of recurrences are not surgically curable, and any resection is likely to be palliative or futile. Most patients with liver metastases are not candidates for resection and the survival rate after liver resection is very low and treatments for peritoneal metastases are still investigational $[16,17]$.

Chemotherapy is considered the mainstay in the treatment of recurrent gastric cancer and is offered with the aim of improving survival and quality of life. Several randomized trials have indicated that patients with recurrent gastric cancer generally live longer when treated with chemotherapy than with optimal supportive care [18-20]. Recent advances in chemotherapy have achieved considerable tumor regression with the median overall survival time reported to be between 6 and 13 months [21].

To date, these results notwithstanding, there is no clear evidence that treatment of a recurrence detected at an earlier stage improves outcome.

\section{Quality of Life Issues}

Follow-up is also important to evaluate the side effects of gastric surgery. Gastric resection leads to radical changes in the anatomy and physiology of the gastrointestinal tract and can cause severe nutritional complications.

Feeding problems occur in approximately $30 \%$ of patients, but severe symptoms are present only in $1-2 \%$. The main symptoms are early postprandial satiety, loss of appetite, alteration of taste, reflux, dyspepsia, nausea and/or vomiting, and diarrhea. There are various 'postgastrectomy syndromes' depending on the type of surgery (total or subtotal gastrectomy) and reconstruction technique (BI, BII, or Roux-en-Y). Gastric reservoir dysfunction (dumping syndromes), afferent and efferent loop syndromes, Roux-en-Y stasis syndrome, and bile reflux can lead to a reduction of food intake and occasionally to severe malnutrition [22].

Along with feeding concerns after gastric resection, regardless of the reconstruction technique, three metabolic and nutritional disorders may occur: anemia, bone disease, and weight loss due to malabsorption.

Nearly $30 \%$ of patients present microcytic anemia (iron deficiency anemia) or megaloblastic anemia (vitamin $B_{12}$ deficiency). Iron deficiency is the most common anemia following gastric resection. After gastric resection, both acid and pepsin, which are needed for iron absorption, are reduced. Moreover, owing to a lack of intrinsic factor secretion, vitamin $B_{12}$ deficiency is common after gastric resection (mostly after total gastrectomy). In- tramuscular injection of vitamin $\mathrm{B}_{12}$ every $3-4$ months is recommended as a standard treatment for patients with vitamin $\mathrm{B}_{12}$ deficiency after total gastrectomy even if daily oral replacement therapy provides a safe and effective alternative treatment [23].

Bone disease, such as osteoporosis, osteopenia, and ostemalacia, is commonly reported in gastrectomy patients. Its etiology appears to be a combination of decreased intake of calcium, vitamin D, and lactose-containing foods, coupled with altered absorption and metabolism. Symptoms of osteoporosis may develop 10 or more years after gastric surgery because of the large amount of calcium that is normally stored in bone [24].

Weight loss is a frequent finding after gastric surgery and is often temporary. Malabsorption is related to the accelerated passage of a large bolus in the jejunum, vagal denervation which increases the rapidity of the orocecal transit, and bacterial overgrowth due to the decrease in gastric acid secretion, and pancreatic insufficiency [25, 26].

\section{Components of Surveillance}

The main components of a surveillance strategy are office visits for postoperative history and physical examination, blood tests (especially tumor markers), imaging, and endoscopic studies.

There is no consensus regarding the follow-up plan after gastrectomy for cancer, and the optimum modality for the diagnosis of early recurrence is indeed unclear. Although there are many tools to detect recurrent disease in addition to clinical examination (laboratory tests, imaging, and endoscopy), none has high tumor specificity.

\section{Laboratory Tests}

The dosage of serum tumor markers is commonly used during postoperative follow-up because their positivity is easily measured with a simple blood test, but it is well known that they are not specific and cannot localize the recurrence site. CEA and CA19-9 are known to be elevated in the serum of patients with advanced gastric cancer, and the monitoring of CEA and CA19-9 monitoring has been used for the early detection of recurrence after operation for advanced gastric cancer.

In prospective studies, both tumor markers were useful indicators of recurrence, especially in almost all the patients who showed high preoperative levels of these markers $[27,28]$. CA19-9 may be especially useful as a marker for peritoneal recurrence of gastric cancer, and 
CEA for recurrence in the liver [29]. Other tumor markers, such as CA 72-4 and CA125, have been investigated, but their sensitivity is significantly lower than that of CEA and CA19-9 [30].

\section{Imaging}

Reports on the use of imaging in detecting recurrent gastric cancer are few and often limited to descriptions of typical findings. Contrast-enhanced abdominal computed tomography (CT) is used most frequently and is regarded as the most reliable method for assessing cancer recurrence, with a reported accuracy of 60-70\% [31]. To date, however, only few reports have been published on CT findings after gastrectomy. A CT scan has limited value in the distinction of postoperative morphologic changes from tumor recurrence and has a low positive predictive value to detect peritoneal and distant lymph node metastasis [32].

Positron emission tomography (PET) is often useful for detecting different patterns of recurrence, such as local recurrence involving the stomach remnant, regional lymph nodes, peritoneal dissemination, liver metastases, and remote metastases. PET is an advantageous imaging tool because it enables the evaluation of the entire body at once, although PET has limitations such as frequent false-negative cases in early cancer and signet-ring cell tumors and poorly differentiated histotypes. PET is useful when conventional imaging is equivocal, as it can confirm the presence of true recurrence [33].

PET represents the most useful noninvasive imaging modality for the detection of hepatic metastases from gastric cancer with a sensitivity of $90 \%$ compared with a sensitivity of 76,72 , and $55 \%$ reported for magnetic resonance imaging, CT, and ultrasonography, respectively [34-36].

An integrated PET/CT scan provides fusion images, combining functional and anatomic imaging together. This modality has a diagnostic accuracy ranging from 75 to $97 \%$. A PET/CT scan has the greatest utility in patients with a suspicion of recurrences based on tumor marker tests and findings of other imaging modalities [37-39].

All imaging studies have a low accuracy in detecting peritoneal disease, which represents one of the most frequent and feared patterns of recurrence. Barium enema has been used in the diagnosis of peritoneal carcinomatosis in colorectal cancer [40], and this imaging has been used in Japanese institutions to confirm the presence of peritoneal disease when clinically suspected [9].

A recent study by Inoue et al. [41] evaluated the feasibility and accuracy of second-look laparoscopy for pa- tients with gastric cancer at high risk of peritoneal recurrence after completion of 6 months of systemic adjuvant chemotherapy. In this study, second-look laparoscopy was a safe and effective approach for early reassessment of peritoneal disease for the selection of patients who needed further systemic chemotherapy.

\section{Endoscopy}

The use of endoscopy during the follow-up period is recommended when there is a risk of recurrence in the stomach remnant in two cases: after a subtotal gastrectomy and after endoscopic treatment for early gastric cancer. After total gastrectomy endoscopy, it is mostly useful to detect surgical complications like a benign stricture [42].

Lifelong annual follow-up endoscopy is recommended after partial gastrectomy. Two thirds of the patients destined to develop a second primary gastric cancer will show signs of disease within 10 years after surgery. The risk is higher in patients with multiple lesions at initial surgery and in patients with undifferentiated-type carcinoma [43].

Careful endoscopic examination of the entire stump, particularly around the lesser curvature and posterior wall, is essential. Elevated and depressed mucosal changes should be examined histologically. Follow-up endoscopy seems important for the early diagnosis of the second primary. When detected at an early stage, treatment provides excellent disease-free survival. However, when a second primary is detected at a later stage $(\geq \mathrm{T} 2)$, the prognosis is poor even after curative resection [44-46].

After endoscopic treatment by submucosal dissection of early gastric cancer, patients are at high risk for synchronous or metachronous multiple gastric cancers. A large multicenter retrospective cohort study indicated that the incidence rate of synchronous cancer was $9 \%$, that about $20 \%$ of synchronous cancers were missed, and that the annual incidence of metachronous cancer was $3.5 \%$. With an annual follow-up examination, almost all multiple lesions could be treated by endoscopic resection [47].

\section{Evidence from Follow-Up Studies}

All of the medical literature reviews to date have failed to show high-level evidence about any follow-up schedule for curatively resected gastric cancer patients. All data are retrospective and observational, thus preventing any definitive conclusion. 
We selected six studies and one systematic review concerning follow-up after surgery for gastric cancer. All these studies focused on the possible survival benefit of early detection of recurrence by intensive postoperative surveillance. Three studies indicated that an intense postoperative follow-up protocol was successful in identifying asymptomatic recurrences earlier than symptomatic recurrences. Nevertheless, they could not achieve any evident advantage in overall survival $[9,48,49]$.

With this purpose, a study from Memorial Sloan-Kettering Cancer Center [50] showed that follow-up did not detect asymptomatic recurrences earlier than symptomatic recurrences in patients with gastric cancer who underwent a curative gastrectomy. In that report, patients with asymptomatic recurrences showed better postrecurrence and disease-specific survival than those with symptomatic recurrences. In their conclusions, the authors indicated that symptomatic and asymptomatic recurrence patterns are biologically different and associated with different survival outcomes. Similarly in a paper by Kim et al. [31], median overall survival and postrecurrence survival were worse for patients with a symptomatic recurrence than for those with an asymptomatic recurrence. Moreover, in this study, multivariate analysis revealed that the presence of a symptomatic recurrence and disease-free interval were independent prognostic indicators for postrecurrence survival. Furthermore, asymptomatic patients had more benefit from re-resection and postrecurrence chemotherapy; at multivariate survival analysis the presence of symptoms was the only independent factor of poor survival, suggesting a more biologically aggressive disease in symptomatic patients. Bilici et al. [51], in a study on 173 patients with recurrent gastric cancer, found that symptomatic recurrence is an important prognostic factor for postrecurrence survival and that the presence of symptoms may be considered a marker of biologic tumor aggressiveness, which is an important determinant of survival at the time of recurrence diagnosis during follow-up for gastric cancer.

A recent systematic review by Cardoso et al. [52] reviewed five studies enrolling a total of 810 patients and assessing outcomes of follow-up after gastrectomy for gastric cancer. They did not find any evidence suggesting that postoperative surveillance has any survival benefit; it is also stressed that no studies addressed quality of life issues. Major limitations in the review were the study design and a lead-time bias in which the observed prolonged survival is due to earlier detection of recurrence, rather than to an effect on disease outcome.

\section{International Guidelines Review and Expert Interview}

The lack of evidence of follow-up is revealed by the fact that most leading scientific societies and cooperative groups propose different schedules and that many centers apply a follow-up program dictated by past common practices in their medical center. Although guidelines are generally supposed to be based on strong evidence (therefore valid and unbiased), to date they have been based on low level evidence or no evidence at all.

\section{International Guidelines Review}

The American Society of Clinical Oncology (ASCO) does not provide formal guidelines or recommendations for follow-up after gastrectomy for cancer. Similarly, the Japanese Gastric Cancer Association (JGCA) guidelines offer no guidelines on follow-up.

The National Cancer Comprehensive Network (NCCN) guidelines include for all patients a complete history and physical examination every 3-6 months for 1-2 years, every 6-12 months for 3-5 years, and annually thereafter. Other investigations should be made if clinically indicated. Patients who have undergone surgical resection should be monitored and treated as indicated for vitamin $\mathrm{B}_{12}$ and iron deficiency [2].

The European Society of Medical Oncology (ESMO) recommends symptom-driven visits. When symptoms occur, a complete history with physical examination and blood tests should be performed. Other investigations are required only in patients who are candidates for treatment [1].

The Association of Upper Gastrointestinal Surgeons of Great Britain and Ireland, the British Society of Gastroenterology, and the British Association of Surgical Oncology agree that regular review may identify early recurrence, but there is no evidence for specific investigations or that follow-up can affect overall survival. Endoscopy, cross-sectional imaging, and tumor markers have all been evaluated, but lack specificity or sensitivity [53].

The GIRCG (Italian Group for Research on Gastric Cancer) has proposed three different follow-up schedules (mild, moderate, or intensive) after gastrectomy for cancer in relation to a risk score calculated for an individual patient. A logistic regression model is used for the computation of the score; the coefficient $\mathrm{Z}$ is calculated as $\mathrm{Z}=$ $-3.888-0.339$ (middle third) +0.917 (upper third) + 6.266 (diffuse location) +0.027 (age) $+1.075(\mathrm{pT} 2)+$ $2.013(\mathrm{pT} 3-\mathrm{T} 4)+1.668(\mathrm{pN} 1)+3.056(\mathrm{pN} 2)+4.971(\mathrm{pN} 3)$ $-0.848(\mathrm{D} 2-\mathrm{D} 3$ dissection). The value of parametric vari- 
ables was 0 (negative) or 1 (positive), whereas age was considered as a continuous variable. For each patient, the value of the coefficient $\mathrm{Z}$ obtained was included in the formula: $\left(\mathrm{e}^{\mathrm{Z}} / 1+\mathrm{e}^{\mathrm{Z}}\right) \times 100$, which gives risk values ranging from 0 to $100 \%$ [54].

For patients with mild risk ( $<10 \%$ or patients over 80 years), they propose ultrasound of the abdomen and tumor marker assay every 6 months, endoscopy and chest $\mathrm{X}$-ray annually, and a CT scan in case of clinical suspicion or increased levels of tumor markers. For patients with moderate risk (between 10 and 50\%), they propose tumor markers be investigated every 3 months, abdominal ultrasound after 6,18, and 30, and CT scan and endoscopy annually. For patients with high risk $(>50 \%)$, they propose tumor markers every 3 months, CT scan every 6 months, and endoscopy annually. After 5 years of annual clinical monitoring, other exams if clinically indicated should be performed as well as any screening for a second cancer (occult blood test, mammography, PSA, etc.). It should be noted that no evaluation of nutritional or quality of life issues is considered in international guidelines.

\section{Expert Interviews}

A questionnaire was sent out to a selected group of world-renowned experts in the field of surgical oncology. From August to October 2012, the survey was performed via electronic mail. The questionnaire was composed of two sections. The first section was an introductory covering letter in which the purpose of the survey was indicated. The second section made up the main portion of the survey, and asked about follow-up schedules and methodologies. Most questions were yes/no or multiple choice, with several text boxes which allowed for comments from participants to provide additional information or clarification. Six out of 16 questionnaires were returned (table 1). All respondents reported having a strategy for surveillance after surgery for gastric cancer, but there was variance in strategy.

First of all, we asked about the main reason for followup. The primary aim of the follow-up schedule for almost all respondents (4/6) is the evaluation of complications associated with surgery and quality of life issues, and most of them perform nutritional assessment at visits. In one institution (University Hospital of Lille, France), the primary aim is the early detection of recurrence, and another institution's primary aim (Jagiellonian University, Krakow, Poland) is the collection of outcome data for treatment evaluation and/or research purposes.

In four of the six of responders, a follow-up schedule is carried out by a multidisciplinary team (surgeons with medical oncologists) that perform follow-up in their hospitals. In two institutions the follow-up is performed by the surgical team.

No significant differences were reported in terms of follow-up frequency for different disease stages. On average, advanced gastric cancer patients are followed-up every 3 months in the first year postoperatively, as opposed to follow-up every 6 months for early gastric cancer during the first year postoperatively. From the second to fourth postoperative year, the patients were usually seen every 6 months. In all cases, follow-up ends at 5 years after surgery.

Table 1 summarizes the follow-up schedules as reported by respondents. Almost all respondents considered a CT scan as mandatory for detection of all types of recurrence and a PET scan as optional.

One respondent left the question blank because he did not have a systematic follow-up schedule and performs advanced imaging and/or endoscopy during follow-up when symptoms or clinical suspicion of recurrence arise.

\section{Conclusion}

The reported international variations in guidelines for surveillance among follow-up schedules reflects a complete lack of an established body of evidence. Consequently, most recommendations aiming to detect early recurrence of the disease often avoid details on the mode, duration, and intensity of surveillance since they cannot be based on studies with high levels of evidence. Moreover, quality of life issues are omitted in the current literature on surveillance, even though most experts underline the importance of this peculiar subject especially after total gastrectomy.

On the other hand, there is almost no doubt that from most patients' and physicians' perspectives, a good clinical practice should not disregard some kind of postoperative surveillance. However, based on a review of the literature and interviews of experts, we found that routine follow-up of gastric cancer patients is nothing more than a common behavior that is (at least) justified by data collection and outcome auditing in addition to ethical-psychological reasons concerning the anxiety of patients regarding full and prompt information about the evolution of their disease.

Although retrospective series have clearly demonstrated that early diagnosis of tumor recurrence in the asymptomatic phase has not resulted in any evident survival benefit compared to a later symptom-driven diagnosis, 


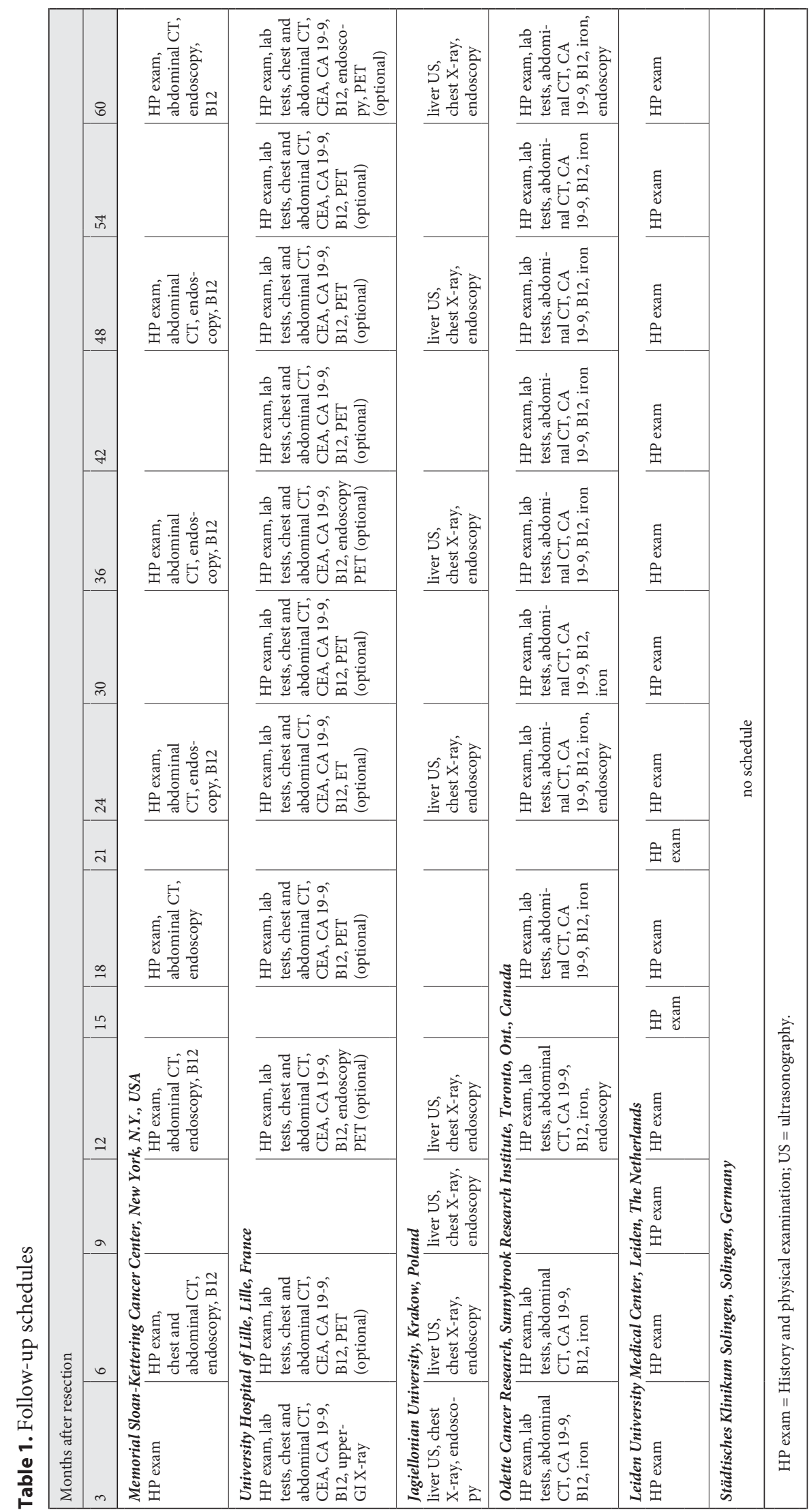


the majority of the centers with a considerable gastric cancer caseload and high level of care apply a policy of clinical and instrumental surveillance with the aim of a timely diagnosis of tumor recurrence and to minimize the nutritional sequelae of gastrectomy. The rationale for this relies on three factors:

(1) The distinct feeling that being subjected to seriated scheduled checks does not represent a source of stress for many patients, but rather has the potential of reassuring them.

(2) The hope that biomedical research will soon offer therapeutic weapons for the metastatic and/or relapsed patients, with results similar to those currently available for patients with colorectal cancer.

(3) The process of improving the standard of quality in surgical oncology cannot be separated from a daily evaluation of the results of therapies by comparing these results between different surgical schools and different patterns of complementary therapies, and this evaluation is made possible only by reliable data on recurrence and survival.

Follow-up schedules based on more solid ground are definitely needed, and this should be done by identifying the tests and examinations with the best reliability and sensitivity and by limiting them to a period in which recurrence is likely.

Surgical oncologists could speculate that patients may receive some benefit by postoperative surgical surveillance if early detection of recurrence leads to any proven survival advantage and/or increased quality of life. Whether there is a preclinical phase in which early detection of recurrence can improve outcome (implying that followed-up patients may have better overall outcomes than unscreened) represents a question apparently suitable for a randomized controlled trial, which is commonly considered as the most rigorous method of determining whether a cause-effect relationship exists between an intervention and its outcome [55]. Although a large randomized trial could determine whether one recommend- ed follow-up program confers a survival benefit, this is unlikely to be rewarding until effective treatments for most patterns of recurrence are available. In fact, at the moment, clinical trials on the efficacy of surveillance strategies with high-risk patients will be doomed to show no efficacy if survival is their primary endpoint because survival after recurrence is poor regardless of the time of diagnosis. At the same time, follow-up strategies in lowrisk patients with good long-term prognosis (i.e. early gastric cancer) necessitate an excessively long time to demonstrate clear improvements in outcome. In both cases, huge sample sizes, money, and time are almost insurmountable obstacles.

Consensus methods are alternative means of dealing with conflicting or scarce scientific evidence. The focus of consensus methods lies where unanimity of opinion does not exist, owing to a lack of scientific evidence or when there is contradictory evidence about an issue. Consensus methods overcome some of the disadvantages normally found with decision-making in groups or committees, which are commonly dominated by one individual or by coalitions. The consensus method attempts to assess the extent of agreement and to resolve disagreement [56]. Currently, in our opinion, an appropriately designed and methodologically based consensus conference may be a proper tool to establish the best way to adequately perform follow-up in gastric cancer patients.

\section{Acknowledgments}

The authors thank all responders to the questionnaire at the institutions that are listed in table 1: Vivian Strong from Memorial Sloan-Kettering Cancer Center (New York, N.Y., USA), Christophe Mariette from University Hospital of Lille (Lille, France), Piotr Kolodziejczyk from Jagiellonian University (Krakow, Poland), Natalie Coburn from Odette Cancer Research (Sunnybrook Research Institute, Toronto, Ont., Canada), Cornelis J.H. van de Velde and Henk H. Hartgrink from Leiden University Medical Center (Leiden, The Netherlands), and Hans-Joachim Meyer from Städtisches Klinikum Solingen (Solingen, Germany).

\section{References}

1 Okines A, Verheij M, Allum W, Cunningham D, Cervantes A: Gastric cancer: ESMO clinical practice guidelines for diagnosis, treatment and follow-up. Ann Oncol 2010;21 (suppl 5):v50-v54.

-2 Ajani JA, Barthel JS, Bekaii-Saab T, Bentrem DJ, D’Amico TA, Das P, Denlinger C, Fuchs CS, Gerdes H, Hayman JA, Hazard L, Hofstetter WL, Ilson DH, Keswani RN, Kleinberg LR,
Korn M, Meredith K, Mulcahy MF, Orringer MB, Osarogiagbon RU, Posey JA, Sasson AR, Scott WJ, Shibata S, Strong VE, Washington MK, Willett C, Wood DE, Wright CD, Yang G, NCCN Gastric Cancer Panel: Gastric Cancer. J Natl Compr Canc Netw 2010;8:378409.

- 3 Renehan AG, Egger M, Saunders MP, O'Dwyer ST: Impact on survival of intensive follow up after curative resection for colorectal cancer: systematic review and meta-analysis of randomized trials. BMJ 2002;324:813.

4 Smith TJ, Davidson NE, Schapira DV, Grunfeld E, Muss HB, Vogel VG 3rd, Somerfield MR: American Society of Clinical Oncology 1998 update of recommended breast cancer surveillance guidelines. J Clin Oncol 1999;17: 1080-1082. 
5 Jemal A, Bray F, Center MM, Ferlay J, Ward E, Forman D: Global cancer statistics. CA Cancer J Clin 2011;61:69-90.

-6 Shiraishi N, Inomata M, Osawa N, Yasuda K, Adachi Y, Kitano S: Early and late recurrence after gastrectomy for gastric carcinoma. Univariate and multivariate analyses. Cancer 2000;89:255-261.

7 Katai H, Maruyama K, Sasako M, Sano T, Okajima K, Kinoshita T, Naparkov A: Mode of recurrence after gastric cancer surgery. Dig Surg 1994;11:99-103.

8 Kodera Y, Ito S, Yamamura Y, Mochizuki Y, Fujiwara M, Hibi K, Ito K, Akiyama S, Nakao A: Follow-up surveillance for recurrence after curative gastric cancer surgery lacks survival benefit. Ann Surg Oncol 2003;10:898-902.

-9 Whiting J, Sano T, Saka M, Fukagawa T, Katai H, Sasako M: Follow-up of gastric cancer: a review. Gastric Cancer 2006;9:74-81.

$\rightarrow 10$ Eom BW, Yoon H, Ryu KW, Lee JH, Cho SJ, Lee JY, et al: Predictors of timing and patterns of recurrence after curative resection for gastric cancer. Dig Surg 2010;27:481-486.

-11 Yoo CH, Noh SH, Shin DW, Choi SH, Min JS: Recurrence following curative resection for gastric carcinoma. Br J Surg 2000;87:236-242.

12 Salem A, Hashem S, Mula-Hussain LY, Mohammed I, Nour A, Shelpai W, Daoud F, Morcos B, Yamin Y, Jaradat I, Khader J, Almousa A: Management strategies for locoregional recurrence in early-stage gastric cancer: retrospective analysis and comprehensive literature review. J Gastrointest Cancer 2012; 43:77-82.

13 Tersmette AC, Offerhaus GJ, Tersmette KW, Giardiello FM, Moore GW, Tytgat GN, Vandenbroucke JP: Meta-analysis of the risk of gastric stump cancer: detection of high risk patient subsets for stomach cancer after remote partial gastrectomy for benign conditions. Cancer Res 1990;50:6486-6489.

14 Lundegardh G, Adami HO, Helmick C, Zack M, Meirik O: Stomach cancer after partial gastrectomy for benign ulcer disease. N Engl J Med 1988;319:195-200.

15 Nozaki I, Nasu J, Kubo Y, Tanada M, Nishimura R, Kurita A: Risk factors for metachronous gastric cancer in the remnant stomach after early cancer surgery. World J Surg 2010;34:1548-1554.

16 Sakamoto Y, Ohyama S, Yamamoto J, Yamada K, Seki M, Ohta K, Kokudo N, Yamaguchi T, Muto T, Makuuchi M: Surgical resection of liver metastases of gastric cancer: an analysis of a 17-year experience with 22 patients. Surgery 2003;133:507-511.

-17 Jensen EH, Tuttle TM: Preoperative staging and postoperative surveillance for gastric cancer. Surg Oncol Clin N Am 2007;16:329-342.

-18 Murad AM, Santiago FF, Petroianu A, Rocha PR, Rodrigues MA, Rausch M: Modified therapy with 5-fluorouracil, doxorubicin and methotrexate in advanced gastric cancer. Cancer 1993;72:37-41.
Glimelius B, Ekstrom K, Hoffman K, Graf W, Sjodén PO, Haglund U, Svensson C, Enander LK, Linné T, Sellstrom H, Heuman R: Randomized comparison between chemotherapy plus best supportive care with best supportive care in advanced gastric cancer. Ann Oncol 1997;8:163-168.

20 Pyrhoenen S, Kuitunen T, Nyandoto P, Kouri M: Randomized comparison of 5-fluorouracil, epidoxorubicin, and methotrexate (FEMTX) plus supportive care with supportive care alone in patients with non-resectable gastric cancer. Br J Cancer 1995;71:587-591.

21 Japanese Gastric Cancer Association: Japanese gastric cancer treatment guidelines 2010 (ver. 3). Gastric Cancer 2011;14:113-123.

22 Eagon JC, Miedema BW, Kelly KA: Postgastrectomy syndrome. Surg Clin North Am 1992;72:445-465.

23 Kim HI, Hyung WJ, Song KJ, Choi SH, Kim $\mathrm{CB}$, Noh SH: Oral vitamin $\mathrm{B}_{12}$ replacement: an effective treatment for vitamin $B_{12}$ deficiency after total gastrectomy in gastric cancer patients. Ann Surg Oncol 2011;18:37113717.

24 Tovey FI, Hall ML, Ell PJ, Hobsley M: Postgastrectomy osteoporosis. Br J Surg 1991;78: 1335-1337.

25 Tovey FI, Hobsley M: Post-gastrectomy patients need to be followed up for 20-30 years. World J Gastroenterol 2000;6:45-48.

26 Bae JM, Park JW, Yang HK, Kim JP: Nutritional status of gastric cancer patients after total gastrectomy. World J Surg 1998;22:254260.

27 Takahashi Y, Takeuchi T, Sakamoto J, Touge T, Mai M, Ohkura $\mathrm{H}$, et al: The usefulness of CEA and/or CA19-9 in monitoring for recurrence in gastric cancer patients: a prospective clinical study. Gastric Cancer 2003;6:142145.

28 Ikeda Y, Mori M, Kajiyama K, Kamakura T, Maehara Y, Haraguchi Y, Sugimachi K: Indicative value of carcinoembryonic antigen (CEA) for liver recurrence following curative resection of stage II and III gastric cancer. Hepatogastroenterology 1996;43:1281-1287.

29 Choi SR, Jang JS, Lee JH, Roh MH, Kim MC, Lee WS, Qureshi W: Role of serum tumor markers in monitoring for recurrence of gastric cancer following radical gastrectomy. Dig Dis Sci 2006;51:2081-2086.

30 Lai IR, Lee WJ, Huang MT, Lin HH: Comparison of serum CA72-4, CEA, TPA, CA19-9 and CA125 levels in gastric cancer patients and correlation with recurrence. Hepatogastroenterology 2002;49:1157-1160.

31 Kim JH, Jang YJ, Park SS, Park SH, Mok YJ: Benefit of post-operative surveillance for recurrence after curative resection for gastric cancer. J Gastrointest Surg 2010;14:969-976.

32 Kim KW, Choi BI, Han JK, Kim TK, Kim AY, Lee HJ, Kim YH, Choi JI, Do KH, Kim HC, Lee MW: Postoperative anatomic and pathologic findings at CT following gastrectomy. Radiographics 2002;22:323-336.
33 Jadvar H, Tatlidil R, Garcia AA, Conti PS: Evaluation of recurrent gastric malignancy with [F-18]-FDG positron emission tomography. Clin Radiol 2003;58:215-221.

34 Kinkel K, Lu Y, Both M, Warren RS, Thoeni RF: Detection of hepatic metastases from cancers of the gastrointestinal tract by using noninvasive imaging methods (US, CT, MR imaging, PET): a meta-analysis. Radiology 2002; 224:748-756.

35 Stahl A, Ott K, Weber WA, Fink U, Siewert JR, Schwaiger M: Correlation of FDG uptake in gastric carcinomas with endoscopic and histopathological findings. J Nucl Med 2001; 42:78P-79P.

36 Park MJ, Lee WJ, Lim HK, Park KW, Choi JY, Kim BT: Detecting recurrence of gastric cancer: the value of FDG PET/CT. Abdom Imaging 2009;34:441-447.

37 Sun L, Su XH, Guan YS, Pan WM, Luo ZM, Wei JH, Wu H: Clinical role of 18F-fluorodeoxyglucose positron emission tomography/ computed tomography in post-operative follow up of gastric cancer: initial results. World J Gastroenterol 2008;14:4627-4632.

38 Sim SH, Kim YJ, Oh DY, Lee SH, Kim DW, Kang WJ, Im SA, Kim TY, Kim WH, Heo DS, Bang YJ: The role of PET/CT in detection of gastric cancer recurrence. BMC Cancer 2009; 9:73.

-39 Ozkan E, Araz M, Soydal C, Kucuk ON: The role of $18 \mathrm{~F}-\mathrm{FDG}$-PET/CT in the preoperative staging and post-therapy follow-up of gastric cancer: comparison with spiral CT. World J Surg Oncol 2011;9:75.

40 Meyers MA, McSweeney J: Secondary neoplasms of the bowel. Radiology 1972;105: $1-11$.

41 Inoue K, Nakane Y, Michiura T, Yamaki S, Yui R, Sakuramoto K, Iwai A, Tokuhara K, Araki Y, Kim S, Nakai K, Sato M, Yamamichi K, Kwon AH: Feasibility and accuracy of second look laparoscopy after gastrectomy for gastric cancer. Surg Endosc 2009;23:23072313.

42 Lee SY, Lee JH, Hwang NC, Kim YH, Rhee PL, Kim JJ, Paik SW, Rhee JC, Sohn TS, Kim S: The role of follow-up endoscopy after total gastrectomy for gastric cancer. Eur J Surg Oncol 2005;31:265-269.

43 Fujita T, Gotohda N, Takahashi S, Nakagohri T, Konishi M, Kinoshita T: Relationship between the histological type of initial lesions and the risk for the development of remnant gastric cancers after gastrectomy for synchronous multiple gastric cancers. World J Surg 2010;34:296-302.

44 Ohashi M, Katai H, Fukagawa T, Gotoda T, Sano T, Sasako M: Cancer of the gastric stump following distal gastrectomy for cancer. Br J Surg 2007;94:92-95.

45 Greene FL: Management of gastric remnant carcinoma based on the results of a 15-year endoscopic screening program. Ann Surg 1996;223:701-708. 
46 Kikuchi S, Sato M, Katada N, Sakuramoto S, Shimao H, Kakita A, Ohida M, Saigenji K: Efficacy of endoscopic surveillance of the upper gastrointestinal tract following distal gastrectomy for early gastric cancer. Hepatogastroenterology 2003;50:1704-1707.

-47 Kato M, Nishida T, Yamamoto K, Hayashi S, Kitamura S, Yabuta T, Yoshio T, Nakamura T, Komori M, Kawai N, Nishihara A, Nakanishi F, Nakahara M, Ogiyama H, Kinoshita K, Yamada T, Iijima H, Tsujii M, Takehara T: Scheduled endoscopic surveillance controls secondary cancer after curative endoscopic resection for early gastric cancer: a multicentre retrospective cohort study by Osaka University ESD study group. Gut 2012, E-pub ahead of print.

-48 Böhner H, Zimmer T, Hopfenmüller W, Berger G, Buhr HJ: Detection and prognosis of recurrent gastric cancer - is routine followup after gastrectomy worthwhile? Hepatogastroenterology 2000;47:1489-1494.
49 Tan IT, So BY: Value of intensive follow-up of patients after curative surgery for gastric carcinoma: J Surg Oncol 2007;96:503-506.

50 Bennett JJ, Gonen M, D’Angelica M, Jaques DP, Brennan MF, Coit DG: Is detection of asymptomatic recurrence after curative resection associated with improved survival in patients with gastric cancer? J Am Coll Surg 2005;201:503-510.

51 Bilici A, Salman T, Oven Ustaalioglu BB, Unek T, Seker M, Aliustaoglu M, Gezen C, Unek T, Yavuzer D, Unlu M, Gumus $\mathrm{M}$, Yilmaz U: The prognostic value of detecting symptomatic or asymptomatic recurrence in patients with gastric cancer after a curative gastrectomy. J Surg Res 2013;180:e1-e9.

52 Cardoso R, Coburn NG, Seevaratnam R, Mahar A, Helyer L, Law C, Singh S: A systematic review of patient surveillance after curative gastrectomy for gastric cancer: a brief review. Gastric Cancer 2012;15(suppl 1):164-167.
53 Allum WH, Blazeby JM, Griffin SM, Cunningham D, Jankowski JA, Wong R, Association of Upper Gastrointestinal Surgeons of Great Britain and Ireland, the British Society of Gastroenterology and the British Association of Surgical Oncology: Guidelines for the management of oesophageal and gastric cancer. Gut 2011;60:1449-1472.

54 Marrelli D, Morgagni P, de Manzoni G, Coniglio A, Marchet A, Saragoni L, Tiberio G, Roviello F, Italian Research Group for Gastric Cancer (IRGGC): Prediction of recurrence after radical surgery for gastric cancer. A scoring system obtained from a prospective multicenter study. Ann Surg 2005;241:247-255.

55 Sibbald B, Roland M: Understanding controlled trials: why are randomised controlled trials important? BMJ 1998;316:201.

56 Jones J, Hunter D: Consensus methods for medical and health services research. BMJ 1995;311:376-378. 